\title{
Mutirões de cirurgia plástica do Instituto Ivo Pitanguy: Fernando de Noronha
}

\author{
Plastic surgery task force of Ivo Pitanguy Institute: Fernando de Noronha
}

\author{
Pablo Maricevich ${ }^{1}$ \\ EDUARDO NisHimURA ${ }^{1}$ \\ RALF BERGER ${ }^{1}$ \\ FranCESCO MAZZARONE ${ }^{2}$ \\ Ivo Pitanguy ${ }^{3}$
}

Trabalho realizado no Instituto Ivo Pitanguy, Rio de Janeiro, RJ, Brasil.

Artigo submetido no SGP (Sistema de Gestão de Publicações) da RBCP.

Artigo recebido: 9/1/2011 Artigo aceito: 5/2/2011

\begin{abstract}
RESUMO
Fernando de Noronha é um arquipélago formado por 21 ilhas, ocupando uma área de $26 \mathrm{~km}^{2}$. $\mathrm{Na}$ área da Saúde, o arquipélago possui o Hospital São Lucas, um Posto de Saúde vinculado ao Programa de Saúde da Família e um SAMU. A I Semana da Cirurgia Plástica do Instituto Ivo Pitanguy em Fernando de Noronha representou a expansão do trabalho feito na $38^{\mathrm{a}} \mathrm{En}$ fermaria da Santa Casa da Misericórdia para lugares carentes da especialidade. A II Semana foi a continuidade natural deste projeto. $\mathrm{O}$ objetivo foi oferecer à população atendimento em cirurgia plástica com consultas, cirurgias e palestra, com o foco na prevenção, diagnóstico e tratamento do câncer de pele, visto que a população local apresenta grande risco para esta doença. A equipe da primeira Missão contou com a participação de dois instrutores e seis residentes do Instituto Ivo Pitanguy, além de uma dermatologista convidada. Já na segunda Missão, houve a participação de um instrutor do Hospital Oswaldo Cruz (SUS-PE). Todos os casos foram documentados e fotografados. Somando-se a primeira e a segunda Missão, foram realizados 280 atendimentos, sendo 93 consultas sem indicação de tratamento cirúrgico e 187 pacientes operados. Do ponto de vista de Saúde Pública, a I e a II Semana da Cirurgia Plástica do Instituto Ivo Pitanguy representaram uma economia significativa para o Estado de Pernambuco. Além disso, este projeto trouxe uma inestimável experiência de vida a todos aqueles que formaram estas equipes. A possibilidade de ajudar à população com as nossas habilidades e conhecimentos só nos orgulha e enaltece ainda mais a tradição de nossa escola.
\end{abstract}

Descritores: Neoplasias Cutâneas. Cirurgia Plástica. Responsabilidade Social.Participação Social.

\begin{abstract}
Fernando de Noronha is an archipelago formed by 21 islands and has an area of 26 square $\mathrm{km}^{2}$. The main island contains the public hospital São Lucas, a Health Care Center and a SAMU (Mobile Emergency Care Service). The I Week of Plastic Surgery of the Ivo Pitanguy Institute expanded the work performed at the $38^{\text {th }}$ Infirmary of the Holy House of Rio de Janeiro to areas with lack of Plastic Surgery Services. The second mission was the natural continuation of the project. The objective of the project was to offer specialized care in Plastic Surgery with consultation, surgeries, and an educative class, focused in the prevention, diagnostic and treatment of skin cancer to the population, due to its susceptibility to the disease. The first team had two instructors, six residents of the Ivo Pitanguy Institute and a dermatologist. The second mission had an instructor from other service (Oswaldo Cruz Hospital), from the State of Pernambuco. All cases were logged and photographed. The two missions performed 280 consultations, being 93 without the need of surgery and 187 surgeries. The Missions enabled a significant economy to the State of Pernambuco. Beyond it, the missions brought a great life experience for those who performed then. The possibility to help people with our skills and knowledge make us very proud and ennoble the tradition of our school.
\end{abstract}

Keywords: Skin Neoplasms. Surgery, Plastic. Social Responsibility. Social Participation.

1. Médico Residente de Cirurgia Plástica do Instituto Ivo Pitanguy, Rio de Janeiro, RJ, Brasil.

2. Membro Titular da Sociedade Brasileira de Cirurgia Plástica; Presidente do Instituto Ivo Pitanguy, Rio de Janeiro, RJ, Brasil.

3. Professor Titular do Curso de Pós-Graduação em Cirurgia Plástica da Escola Médica de Pós-Graduação da Pontifícia Universidade Católica do Rio de Janeiro e do Curso de Pós-Graduação em Cirurgia Plástica do Instituto de Pós-Graduação Médica Carlos Chagas. Chefe da $38^{\mathrm{a}}$ Enfermaria da Santa Casa da Misericórdia do Rio de Janeiro (SCM-RJ). Membro Titular da Sociedade Brasileira de Cirurgia Plástica. Membro Titular da Academia Nacional de Medicina e da Academia Brasileira de Letras. FACS, FICS, TCBC; Patrono da Sociedade Brasileira de Cirurgia Plástica, Rio de Janeiro, RJ, Brasil. 


\section{INTRODUÇÃO}

O ano de 2010 foi marcado positivamente pelos mutirões realizados pela Sociedade de Cirurgia Plástica em conjunto com as Sociedades Regionais em diferentes estados. Assim foi no Mutirão da Jornada Paulista, em São Paulo-SP, no Mutirão em Belém-PA, no Mutirão da Jornada Sul-Brasileira, em CuritibaPR, e no Mutirão da Jornada Centro-Oeste, em Goiânia-GO. Infelizmente, é fato que a população que depende do SUS tem muita dificuldade em conseguir atendimento e realizar cirurgias na nossa especialidade. Isto é uma verdade em todo o território nacional, onde, na grande maioria das vezes, existe uma demanda maior do que a capacidade de atendimento na Cirurgia Plástica dos hospitais públicos.

Além disso, a cirurgia plástica não figura como prioridade no julgamento dos gestores da saúde pública, por não ser, na maioria das vezes, uma cirurgia vital para o paciente, como uma vítima de trauma ou um paciente oncológico. A realização desses mutirões proporciona a esses pacientes da rede pública a possibilidade de atendimento em Cirurgia Plástica de uma forma bem prática e objetiva, onde o tratamento cirúrgico é realizado logo em seguida da consulta ambulatorial, resolvendo o problema o mais rápido possível e desafogando a fila do SUS, o que trará esta mesma oportunidade para outros.

O Instituto Ivo Pitanguy também colaborou neste sentido no ano de 2010, com a realização da I e II Semana da Cirurgia Plástica do Instituto Ivo Pitanguy, em Fernando de Noronha. A iniciativa de realizar I Semana da Cirurgia Plástica do Instituto Ivo Pitanguy em Fernando de Noronha, partiu dos residentes da instituição, com apoio imediato de corpo docente, que vislumbraram a possibilidade de expandir o trabalho que já é feito na $38^{\text {a }}$ Enfermaria da Santa Casa da Misericórdia para lugares que são carentes no atendimento da especialidade. A II Semana da Cirurgia Plástica do Instituto Ivo Pitanguy em Fernando de Noronha representou a continuidade deste projeto.

O sucesso e o impacto social da primeira missão fez com que sua continuidade fosse uma coisa natural e de interesse de ambas as partes.

Em relação ao local dos nossos mutirões, Fernando de Noronha é um arquipélago formado por 21 ilhas e ilhotas, ocupando uma área de $26 \mathrm{~km}^{2}$, situado no Oceano Atlântico, a leste do estado do Rio Grande do Norte. Constitui um Distrito estadual de Pernambuco desde 1988, quando deixou de ser um território federal. É gerida por um administrador-geral designado pelo governo do estado. A ilha principal, chamada de Fernando de Noronha, é a única habitada, tem $17 \mathrm{~km}^{2} \mathrm{e}$ fica a $545 \mathrm{~km}$ do Recife e a $360 \mathrm{~km}$ de Natal. A sua população atualmente é de 3108 habitantes (est. IBGE/2009), com um fluxo médio mensal de 2000 turistas. A maior parte do arquipélago é designada Parque Nacional Marinho (PARNAMAR), uma unidade de conservação administrada pelo Instituto Chico
Mendes de Conservação da Biodiversidade (ICMBio), e o restante, onde existe ocupação humana, é considerado Área de Proteção Ambiental (APA).

O turismo é a principal fonte de renda do arquipélago, que é considerado um dos melhores pontos de mergulho e surfe do Brasil. Na área da Saúde, o arquipélago possui um hospital público, o Hospital São Lucas, um Posto de Saúde vinculado ao Programa de Saúde da Família (PSF) e um Serviço de Atendimento Móvel de Urgência (SAMU). No Posto de Saúde trabalham um médico generalista e um dentista. Já o Hospital São Lucas, que é um hospital de média complexidade, possui um médico generalista, um pediatra, um ginecologistaobstetra e um dentista. O hospital tem atendimento médico 24 horas por dia e ainda dispõe de um Centro Cirúrgico e uma Sala de Parto, no entanto, estes não são utilizados. Na ausência de atendimento em algumas áreas médicas, periodicamente, são realizadas as Semanas dos Especialistas, objetivando o acesso da população a certas especialidades. De outra maneira, o paciente teria que ser encaminhado pelo médico generalista através de uma TFD (Tratamento Fora de Domicílio), marcar uma consulta com um especialista no serviço de saúde público e deslocar-se até o Recife para o atendimento especializado. Isso tudo custeado pelo estado. Cardiologia, ortopedia, dermatologia, psiquiatria e oftalmologia são exemplos de especialidades que fazem parte da Semana do Especialista.

O objetivo do projeto é oferecer à população do arquipélago de Fernando de Noronha atendimento especializado na área de Cirurgia Plástica, com consultas ambulatoriais, procedimentos cirúrgicos e palestra educativa.

A prevenção, diagnóstico e tratamento cirúrgico do câncer de pele são o foco da nossa atuação, visto que a população da ilha constitui um grupo de risco para esta doença. Entretanto, consultas e cirurgias em cirurgia plástica geral também são realizadas, além de revermos os pacientes operados na nossa $1^{\mathrm{a}}$ Missão.

\section{MÉTODO}

A viabilização do projeto se deu por meio da iniciativa do Instituto Ivo Pitanguy, com o apoio do Governo do Estado de Pernambuco, Administração de Fernando de Noronha, Coordenação de Saúde de Fernando de Noronha e Conselho Distrital de Fernando de Noronha.

Não houve qualquer cobrança de honorários médicos por parte da equipe do Instituto Ivo Pitanguy. No Hospital São Lucas (Figura 1), foram realizados os atendimentos ambulatoriais e as cirurgias. Todo o material cirúrgico e hospitalar foi fornecido pela Administração de Fernando de Noronha (Figura 2). Como o Centro Cirúrgico estava em reforma, adaptamos uma enfermaria para a realização dos procedimentos cirúrgicos (Figura 3). A palestra educativa foi realizada no auditório do PSF. 
A equipe da primeira Missão contou com a participação de dois instrutores e seis residentes do Instituto Ivo Pitanguy, além de uma dermatologista convidada, somando nove participantes (Figura 4). Já a equipe da segunda Missão contou com a participação de dois instrutores, sendo um do Instituto Ivo Pitanguy e outro do Hospital Oswaldo Cruz (SUS-PE), seis residentes do Instituto Ivo Pitanguy, e um dermatologista convidado, somando nove participantes ao total também (Figura 5).

Além das atividades de ambulatório e cirurgias, a equipe ficou responsável pela documentação de todos os pacientes com uma ficha de atendimento confeccionada especialmente para a ocasião (Figura 6), registro fotográfico de todos os pré e pós-operatórios (Figura 7) e palestra educativa (Figura 8 e Tabela 1).

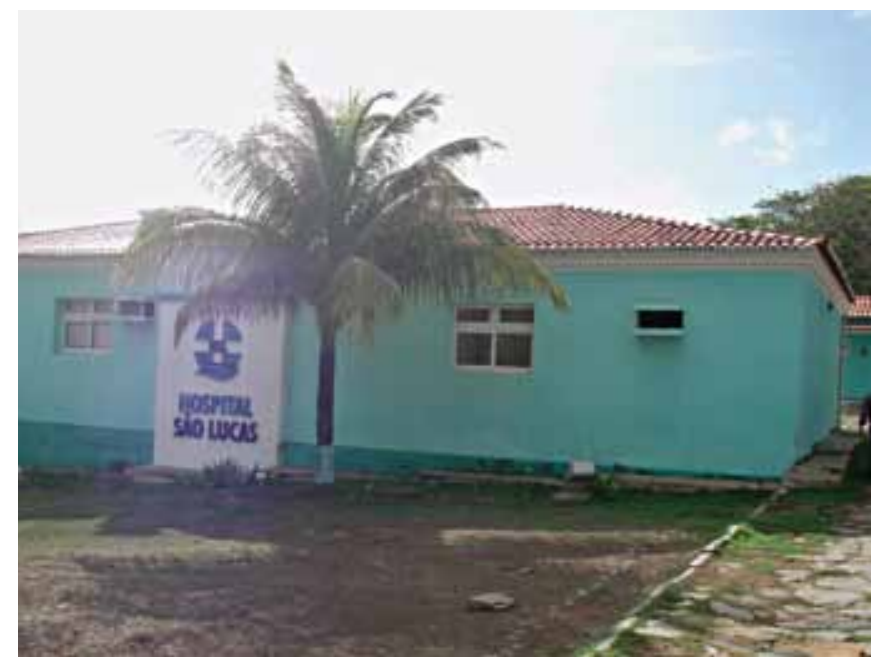

Figura 1 - Hospital São Lucas.

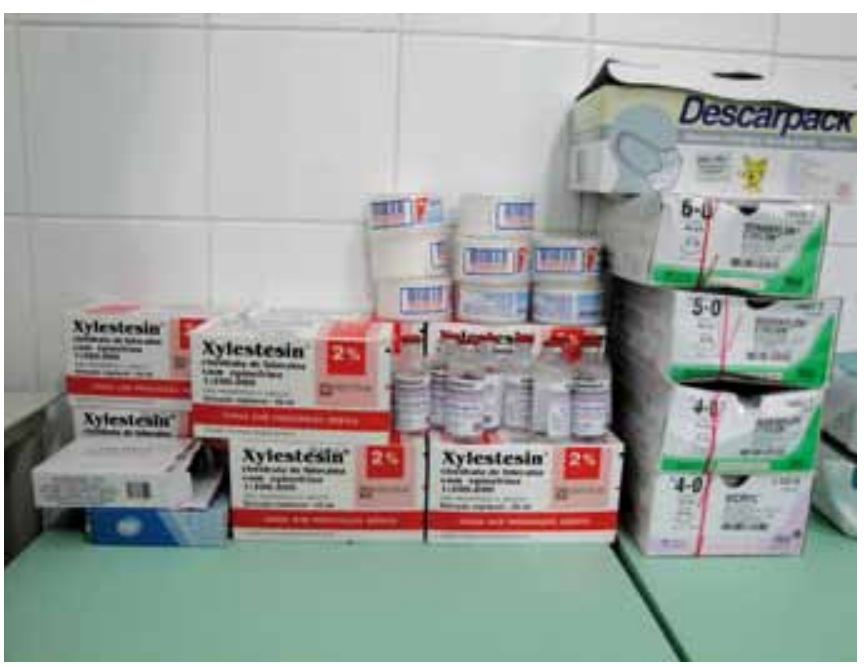

Figura 2-Material cirúrgico e hospitalar.

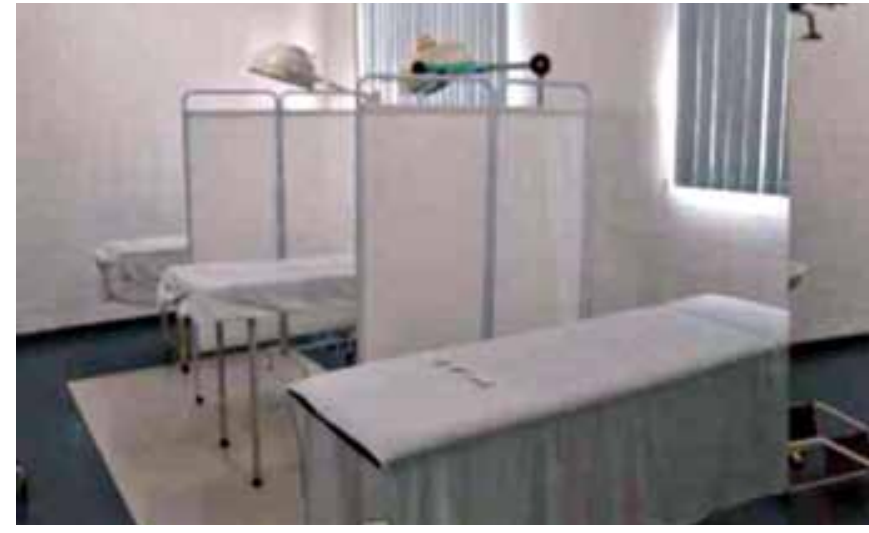

Figura 3 - Centro cirúrgico adaptado na enfermaria.

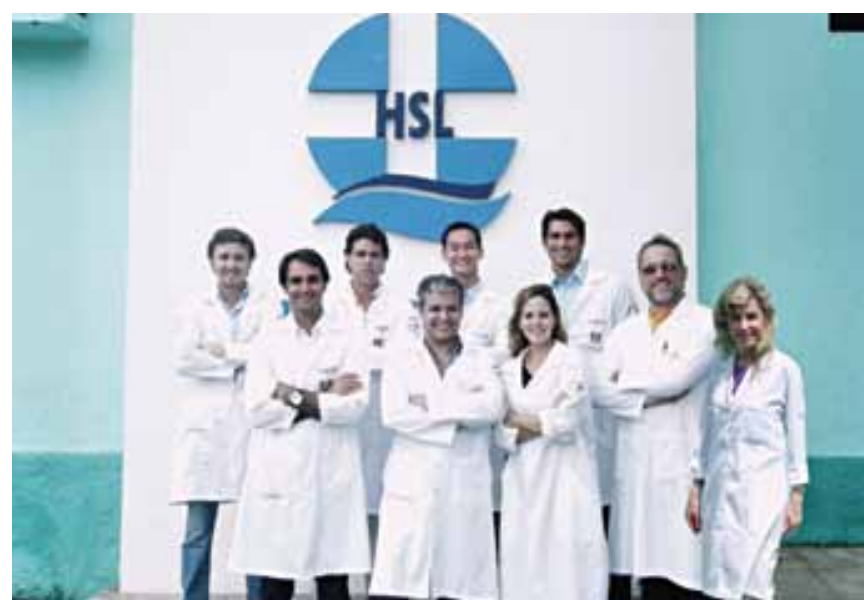

Figura 4 - Equipe da primeira Missão: Tiago Refosco, Conrado d'Avila, Luís Gustavo Prado, Leandro Pereira, Eduardo Nishimura, Gabrielle Wajnberg, Pablo Maricevich, Francesco Mazzarone e Liane Mazzarone.

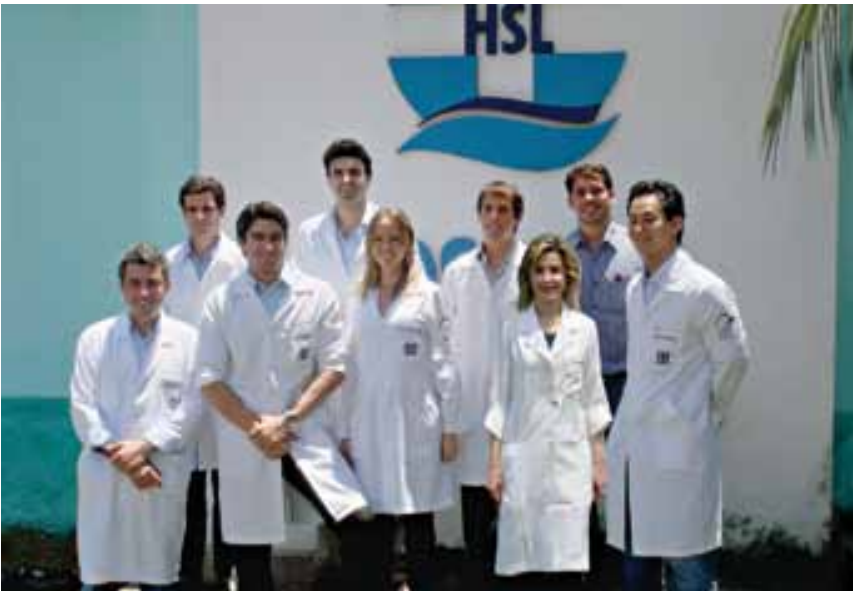

Figura 5-Equipe da segunda Missão: Eduardo Lintz, Ricardo Eisenstein, Pablo Maricevich, Ralf Berger, Franciele Freitas, Rodrigo Duprat, Liane Mazzarone, Rodrigo Quintas e Eduardo Nishimura. 


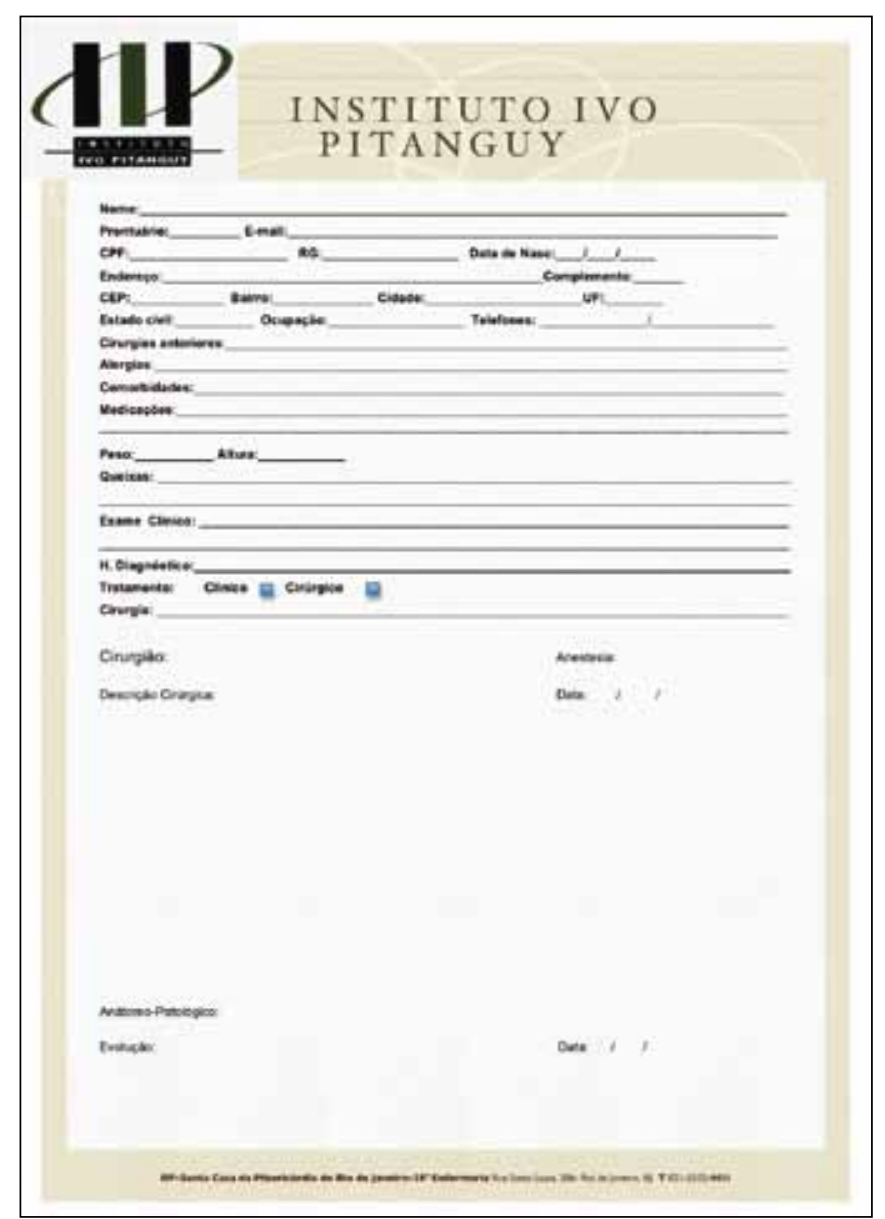

Figura 6 - Ficha de atendimento.

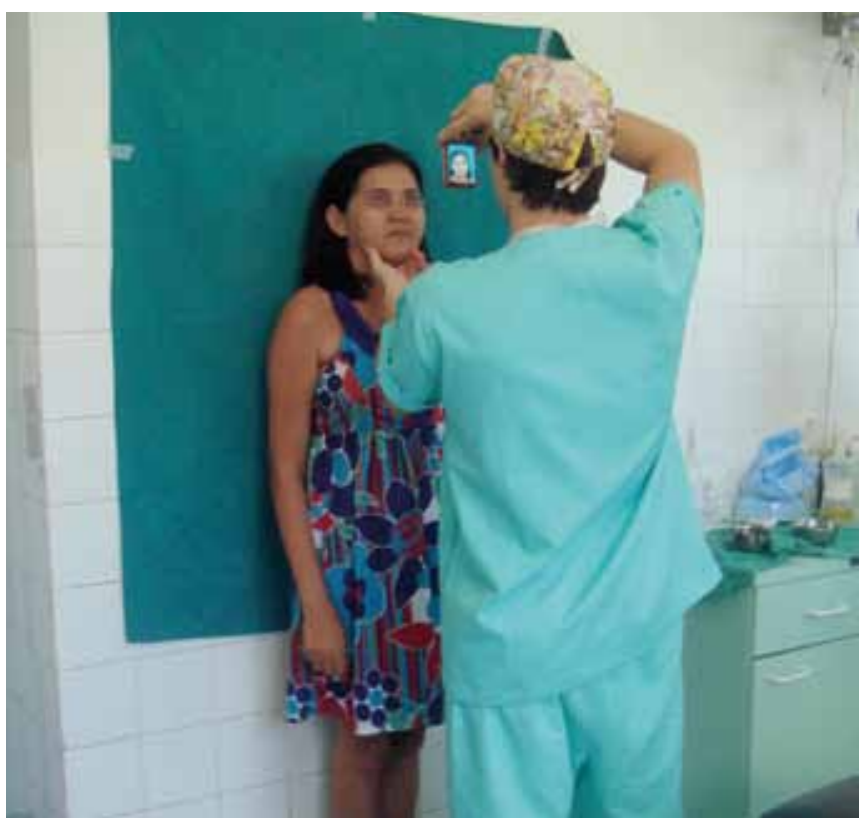

Figura 7 - Documentação fotográfica.

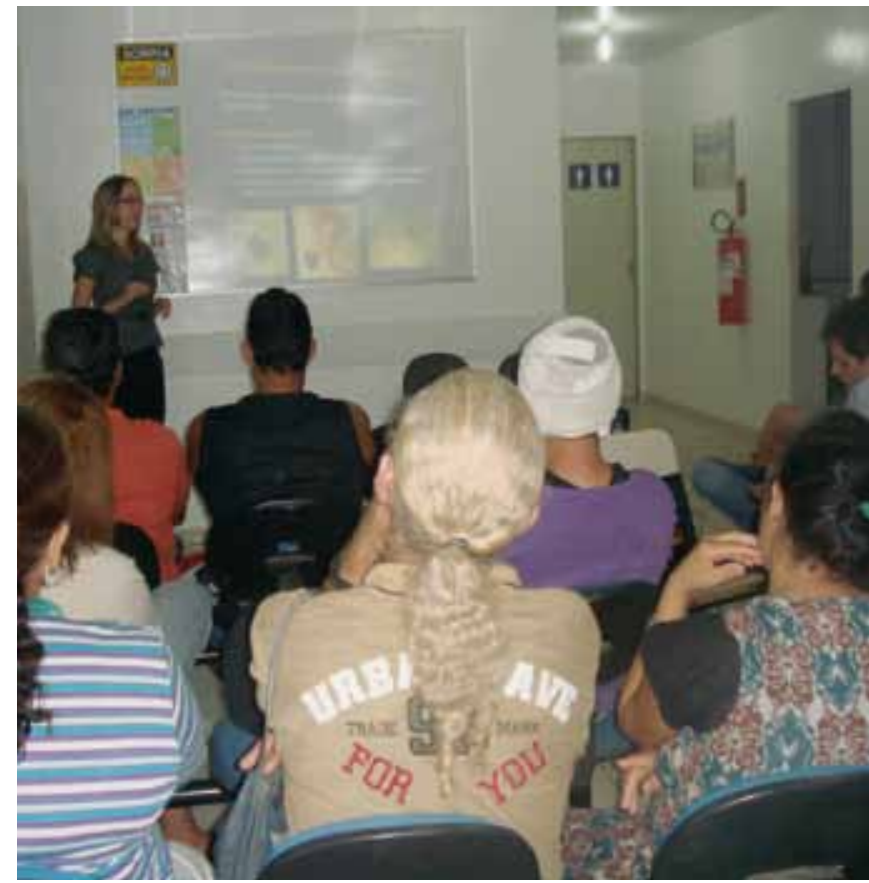

Figura 8-Palestra para a população.

Tabela 1 - Programação do Projeto Noronha.

\begin{tabular}{|c|c|c|c|c|c|}
\hline & Segunda & Terça & Quarta & Quinta & Sexta \\
\hline Manhã & $\begin{array}{c}\text { Ambulatório } \\
\text { Cirurgia }\end{array}$ & $\begin{array}{c}\text { Ambulatório } \\
\text { Cirurgia }\end{array}$ & $\begin{array}{c}\text { Ambulatório } \\
\text { Cirurgia }\end{array}$ & $\begin{array}{c}\text { Ambulatório } \\
\text { Cirurgia }\end{array}$ & $\begin{array}{l}\text { Revisão } \\
\text { cirúrgica }\end{array}$ \\
\hline Tarde & $\begin{array}{c}\text { Ambulatório } \\
\text { Cirurgia }\end{array}$ & $\begin{array}{c}\text { Ambulatório } \\
\text { Cirurgia }\end{array}$ & \begin{tabular}{|c|} 
Ambulatório \\
Cirurgia
\end{tabular} & $\begin{array}{c}\text { Ambulatório } \\
\text { Cirurgia }\end{array}$ & \\
\hline Noite & & Palestra & & & \\
\hline
\end{tabular}

\section{RESULTADOS}

Somando-se a primeira e a segunda Missão, foram realizados 280 atendimentos no total, sendo 93 consultas clínicas, onde não houve tratamento cirúrgico, e 187 pacientes operados.

Nas consultas clínicas, a idade variou de 6 semanas a 36 anos, com idade média de 37,7 anos. O sexo predominante foi o feminino, representando $77 \%$ dos consultados. Durante as consultas, orientamos os pacientes de acordo com o seu diagnóstico e prescrevemos tratamento clínico quando necessário (Figuras 9 e 10).

Alguns pacientes tinham indicação cirúrgica, entretanto a cirurgia não foi realizada porque o paciente não quis ou por julgarmos se tratar de uma cirurgia de porte maior. Quarenta e oito diagnósticos foram dados durante as consultas (Tabela 2). 


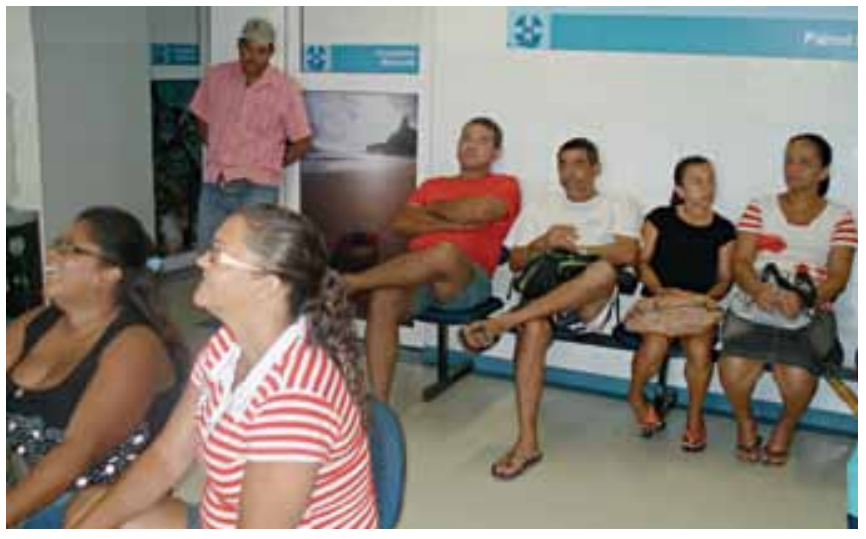

Figura 9 - Sala de espera do ambulatório.

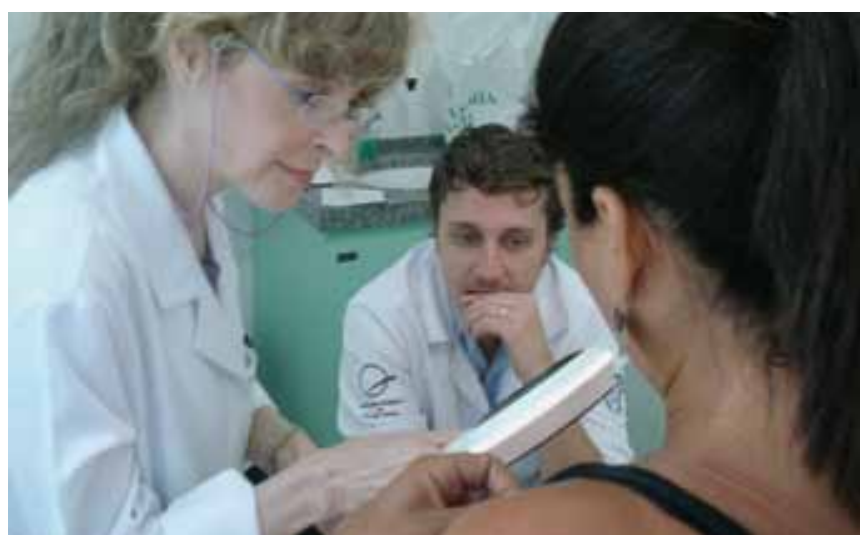

Figura 10 - Consulta ambulatorial.

Nos 187 pacientes operados (Figuras 11A a 11D), a idade variou de 5 a 82 anos, sendo a idade média de 37,8 anos. O sexo predominante também foi o feminino, representando $67,5 \%$ dos operados. A maioria dos pacientes submeteu-se a mais de um procedimento na mesma oportunidade cirúrgica, com média de 2,8 cirurgias por paciente e totalizando 519 procedimentos cirúrgicos realizados (Tabela 3). Todas as lesões suspeitas (91 lesões) foram devidamente enviadas para exame anatomopatológico.

Do ponto de vista de custo em Saúde Pública, a I e a II Semana da Cirurgia Plástica do Instituto Ivo Pitanguy representaram uma economia significativa para o Estado de Pernambuco. Para realizar o mesmo número de atendimentos e operar o mesmo número de pacientes sem a presença da nossa equipe no Arquipélago, o Estado de Pernambuco teria que custear: (1) transporte aéreo do trecho Fernando de Noronha - Recife - Fernando de Noronha; (2) consulta médica especializada; (3) a cirurgia em si. No caso do paciente de Fernando de Noronha, ainda existe um agravante: a Administração da Ilha não se responsabiliza pela acomodação e alimentação do paciente enquanto este se encontra no Recife para Tratamento Fora do Domicílio (TFD), então este é um

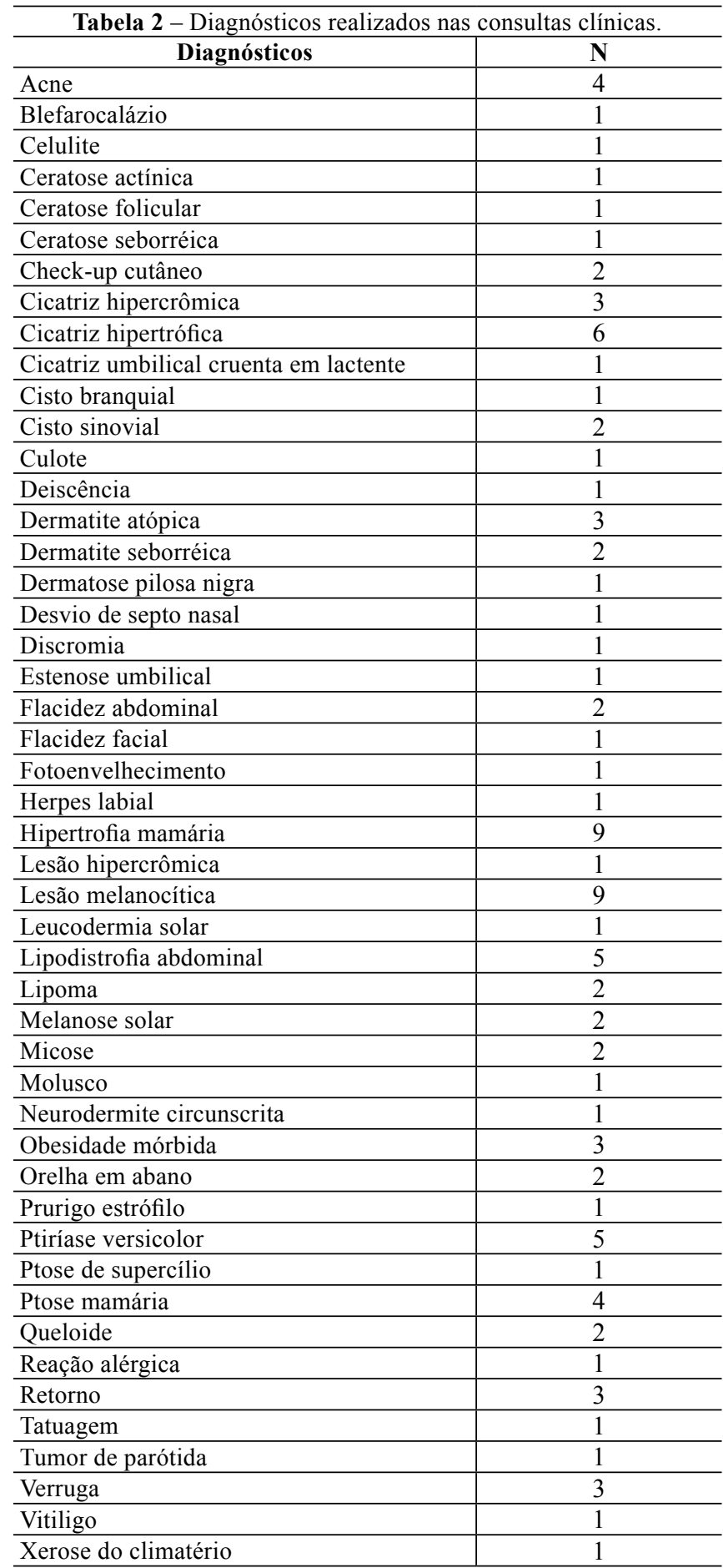

custo adicional que não temos como calcular objetivamente. As Tabelas 4 e 5 simulam estes gastos e são baseadas nas informações obtidas pela Direção do Hospital São Lucas (passagens aéreas) e pelas tabelas atuais do SUS, disponíveis ao público na Internet (http://sigtap.datasus.gov.br). 
Para uma simulação do custo total que o Estado de Pernambuco teria na ausência deste projeto (Tabela 6), utilizamos o número de pacientes operados (187) e não o número de procedimentos cirúrgicos (519), e o cálculo foi feito baseado na cirurgia que fizemos com mais frequência, a excisão e sutura de lesão na pele com retalho.

Obviamente, existiu também um custo para o Estado de Pernambuco (incluindo a Administração de Fernando de Noronha) para receber e dar um suporte a nossa equipe em Fernando de Noronha, nas duas oportunidades que estivemos lá (Tabela 7). Estes gastos foram com: (1) transporte aéreo Recife Fernando de Noronha - Recife e (2) alimentação. Houve isenção da taxa de preservação (TP), assim como é feito para qualquer um que esteja a serviço da Administração e a acomodação, a casa em que ficamos, foi doação de um empresário local.

Então, subtraindo a suposto custo que o Estado de Pernambuco teria para proporcionar o mesmo número de atendimentos e operar a mesma quantidade de pacientes na ausência da nossa equipe em Fernando de Noronha pela real custo das Semanas da Cirurgia Plástica do Instituto Ivo Pitanguy em Fernando de Noronha, nosso projeto representou uma economia de R\$ 166.763,47 aos cofres públicos (Tabela 8).

As Figuras 12 a 16 ilustram alguns casos clínicos selecionados: mamilo invertido, cicatriz em abdome, cisto em face, tumor nasal e orelha em abano.

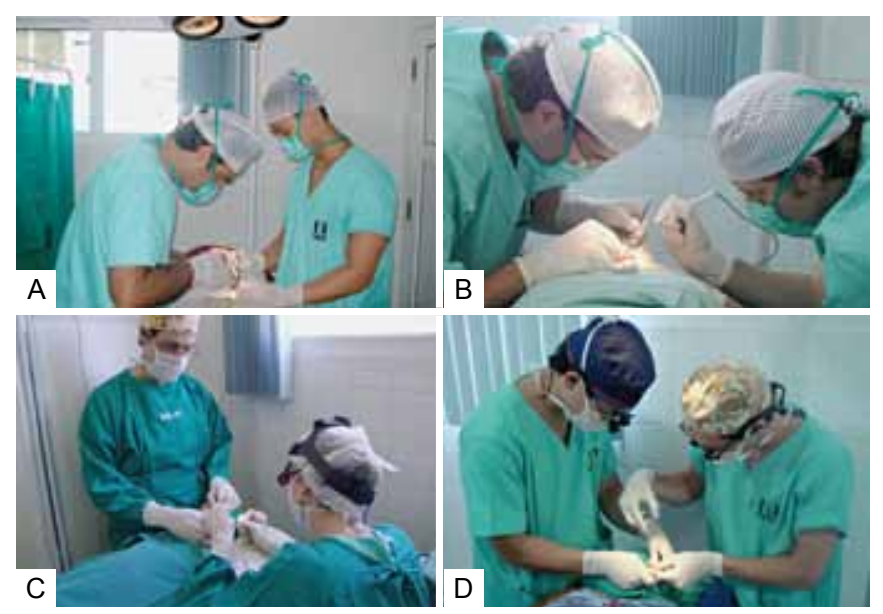

Figura 11 - Pacientes sendo operados.

A

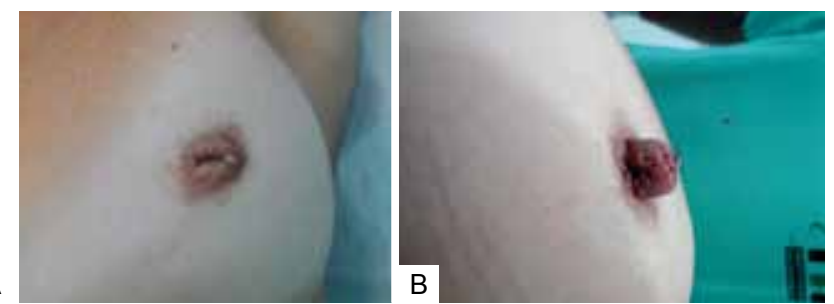

Figura 12 - Caso 1: Mamilo invertido corrigido pela técnica de Pitanguy. A: Pré-operatório; B: Pós-operatório.
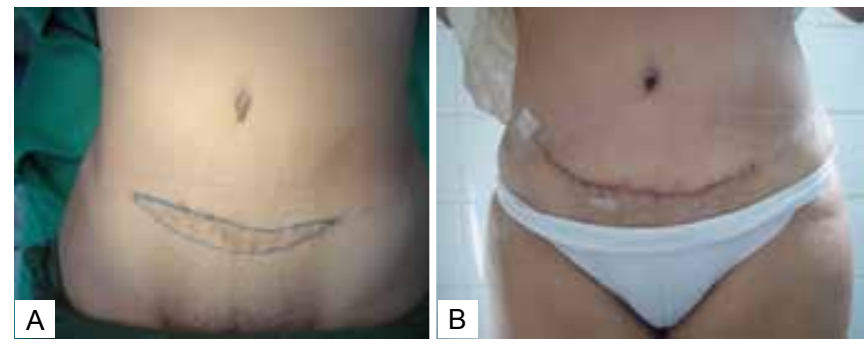

Figura 13 - Caso 2: Ressecção de cicatriz inestética de abdome. A: Pré-operatório; $\boldsymbol{B}$ : Pós-operatório.
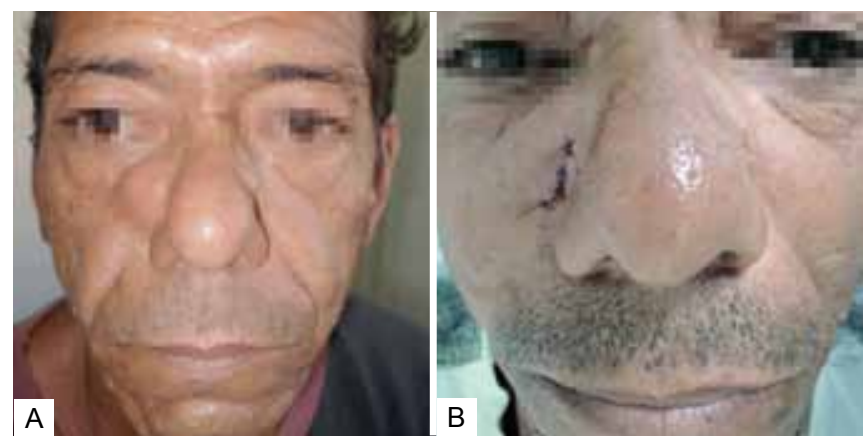

Figura 14-Caso 3: Exérese de cisto em face. A: Pré-operatório; B: Pós-operatório.

\begin{tabular}{l|c}
\hline \multicolumn{1}{c}{ Tabela 3 $\begin{array}{c}\text { Procedimentos cirúrgicos realizados nos } \\
\text { 104 pacientes operados. }\end{array}$} \\
\hline \begin{tabular}{l}
\multicolumn{1}{c}{ Procedimentos cirúrgicos } \\
Abscesso
\end{tabular} \\
\hline Antraz & 4 \\
\hline Ceratoacantoma & 2 \\
\hline Cicatriz inestética & 24 \\
\hline Cisto sebáceo & 35 \\
\hline Deformidade em hélice & 1 \\
\hline Fibroma & 2 \\
\hline Freio lingual & 2 \\
\hline Granuloma & 5 \\
\hline Hemangioma & 5 \\
\hline Hidroadenite & 1 \\
\hline Hipertrofia de mamilo & 2 \\
\hline Lesãomelanocítica $(* 91$ lesões suspeitas enviadas para biopsia) & 377 \\
\hline Lipoma & 7 \\
\hline Lobuloplastia & 10 \\
\hline Mamilo acessório & 1 \\
\hline Mamilo invertido & 5 \\
\hline Milium & 2 \\
\hline Nódulo inflamatório & 2 \\
\hline Otoplastia bilateral & 6 \\
\hline Otoplastia unilateral & 1 \\
\hline Punção de linfonodo & 1 \\
\hline Queloide & 1 \\
\hline Tumor de pálpebra & 1 \\
\hline Verruga & 21 \\
\hline Total & 519 \\
\hline
\end{tabular}




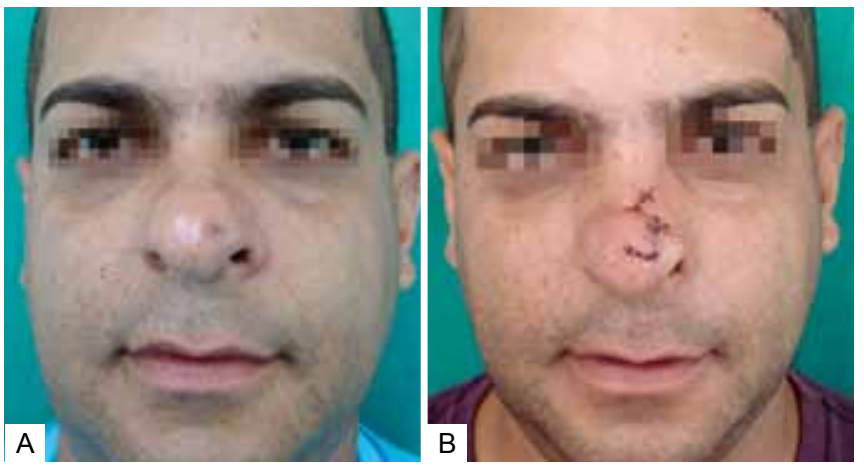

Figura 15 - Caso 4: Tumor de ponta nasal com retalho bilobado. A: Pré-operatório; $\boldsymbol{B}$ : Pós-operatório.

\begin{tabular}{l|c|c}
\hline \multicolumn{1}{c}{ Tabela 4 - Simulação parcial de custo para o Estado de Pernambuco. } \\
\hline \multicolumn{1}{c}{ Item } & Custo por paciente & Custo \\
\hline $\begin{array}{l}\text { Transporte aéreo Noronha - } \\
\text { Recife - Noronha }\end{array}$ & $\mathrm{R} \$ 400,00$ por paciente & $280 \times 400,00=$ \\
\hline $\begin{array}{l}\text { Acomodação e alimentação no } \\
\text { Recife }\end{array}$ & $?$ & $?$ \\
\hline $\begin{array}{l}\text { Consulta médica especializada } \\
\text { (Tabela SUS) }\end{array}$ & $\mathrm{R} \$ 10,00$ por paciente & $280 \times 10,00=$ \\
\hline
\end{tabular}

\begin{tabular}{l|c}
\hline \multicolumn{2}{c}{ Tabela 5 - Cirurgias realizadas. } \\
\hline \multicolumn{1}{c}{ Cirurgia } & Tabela SUS (Reais) \\
\hline Excisão e sutura de lesão na pele com retalho & 356,81 \\
\hline Ressecção de lesão maligna em cabeça e pescoço & 527,65 \\
\hline Tratamento cirúrgico de retração cicatricial & 296,51 \\
\hline Reconstrução de lóbulo de orelha & 391,88 \\
\hline Otoplastia & 391,88 \\
\hline
\end{tabular}

Fonte: http://sigtap.datasus.gov.br

\begin{tabular}{l|c|c}
\hline \multicolumn{3}{c}{ Tabela 6 - Simulação do custo total para o Estado de Pernambuco. } \\
\hline \multicolumn{1}{c|}{ Item } & Custo por paciente & Custo \\
\hline Transporte aéreo & $280 \times 400,00$ & $\mathrm{R} \$ 112.000,00$ \\
\hline Acomodação + alimentação & Paciente & $?$ \\
\hline Consultas especializadas & $280 \times 10,00$ & $\mathrm{R} \$ 2.800,00$ \\
\hline Cirurgias & $187 \times 356,81$ & $\mathrm{R} \$ 66.723,47$ \\
\hline Total & & $\mathrm{R} \$ 181.523,47$ \\
\hline
\end{tabular}

Tabela 7 - Custo Real da Semana da Cirurgia Plástica para o

\begin{tabular}{l|c|c}
\multicolumn{3}{c}{ Estado de Pernambuco. } \\
\hline \multicolumn{1}{c|}{ Item } & Custo por paciente & Custo \\
\hline $\begin{array}{l}\text { Transporte aéreo (Recife } \\
\text { - Noronha - Recife) }\end{array}$ & $2 \times 9 \times 400,00$ & $\mathrm{R} \$ 7.200,00$ \\
\hline Taxa de Preservação & Isento & Zero \\
\hline Acomodação & Doação & Zero \\
\hline Alimentação & $\begin{array}{l}30,00 \text { por refeição (2 refeições por } \\
\text { dia para cada pessoa por 7 dias x 2) }\end{array}$ & $\mathrm{R} \$ 7.560,00$ \\
\hline Total & & $\mathrm{R} \$ 14.760,00$ \\
\hline
\end{tabular}

\begin{tabular}{l|c}
\hline \multicolumn{2}{c}{ Tabela 8 - Economia para o Estado de Pernambuco. } \\
\hline \multicolumn{1}{c}{ Custo } & R\$ \\
\hline $\begin{array}{l}\text { Simulação do custo total para o Estado de } \\
\text { Pernambuco }\end{array}$ & $\mathrm{R} \$ 181.523,47$ \\
\hline Custo real das Semanas da Cirurgia Plástica & $\mathrm{R} \$ 14.760,00$ \\
\hline Economia & $\mathrm{R} \$ 166.763,47$ \\
\hline
\end{tabular}
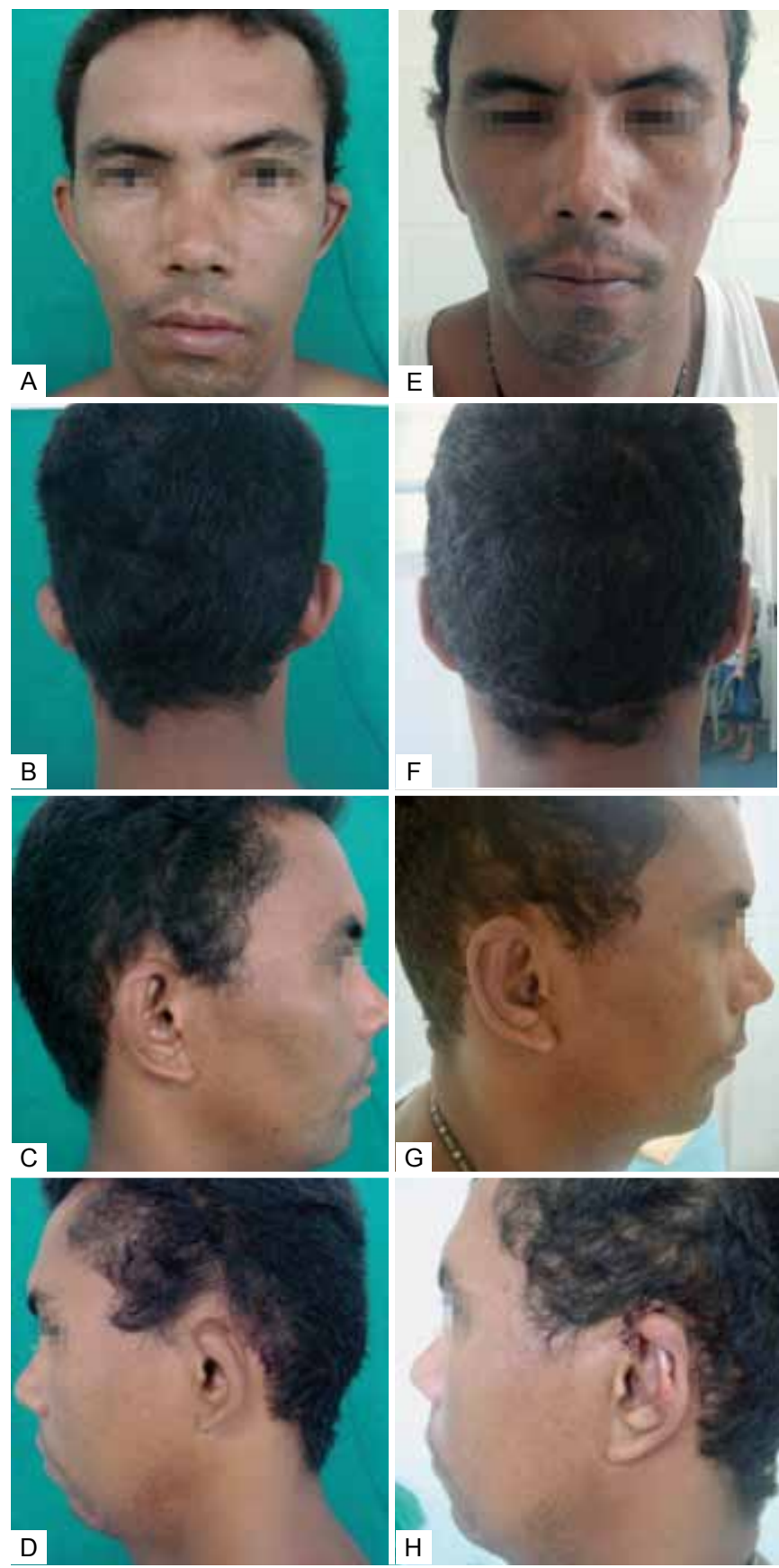

Figura 16 - Caso 5: Orelha em abano à direita e "cup ear" ’̀ esquerda corrigidas pela técnica em ilha de Pitanguy. A, B, C e D: Pré-operatório; $\boldsymbol{E}, \boldsymbol{F}, \boldsymbol{G}$ e $\boldsymbol{H}$ : Pós-operatório. 


\section{DISCUSSÃO}

O Instituto Ivo Pitanguy enxerga de forma muito positiva iniciativas como estas realizadas em 2010. Acreditamos que, além do óbvio benefício social e da evidente contribuição para a saúde pública, movimentos desta razão trazem um grande impacto positivo para a nossa especialidade. A Cirurgia Plástica que, na maioria das vezes, é levada à população leiga pelos meios de comunicação como sinônimo de cirurgia do embelezamento ou, algumas vezes, é capa de jornal quando maus profissionais sem formação se aventuram na especialidade, passa a ser apresentada em outra perspectiva: a cirurgia reparadora capaz de aliviar o sofrimento de pacientes portadores de deformidades que comprometem a qualidade de vida e de diagnosticar e tratar doenças malignas da pele.

Um outro ponto a ser levado em consideração é o compromisso que sentimos, de certa forma, em relação à população que depende do SUS. Muitos de nós tivemos nossa formação médica, em Cirurgia Geral ou em Cirurgia Plástica, realizada em instituições federais, estaduais ou municipais. Assim, em muitos casos, toda ou alguma parte de nossa formação foi custeada pelo Estado. É fato que, durante o nosso período de formação, já prestamos uma grande contribuição ao funcionamento destes serviços de saúde. No entanto, uma vez formados cirurgiões plásticos, realizar mutirões como estes nos proporciona uma sensação muito boa de retribuição ao que nos foi oferecido, tanto ao Estado quanto aos pacientes com os quais aprendemos a fazer as nossas primeiras suturas.

No que diz respeito ao nosso desempenho, a I e II Semana da Cirurgia Plástica do Instituto Ivo Pitanguy alcançaram números significativos. Tanto no que diz respeito à quantidade de pacientes atendidos e operados, quanto no aspecto de economia para o Estado em saúde pública, com um total de 280 atendimentos, 187 pacientes operados e uma economia estimada em R \$166.763,47. Neste âmbito não existe qualquer tipo de competição e toda ajuda é válida, não importando o quanto, mas, a título de comparação, podemos tomar como referência os Mutirões de Belém, Curitiba e Goiânia, que tiveram, respectivamente, 31,33 e 10 pacientes operados. $\mathrm{O}$ Mutirão de São Paulo realizado durante a Jornada Paulista teve a participação de 30 hospitais, no entanto, não teve o número de pacientes operados divulgado. Não há registros de economia estimada destes projetos para os cofres públicos. Todos estes Mutirões foram realizados em 2010.

\section{CONCLUSÃO}

Acreditamos que a I e a II Semana da Cirurgia do Instituto Ivo Pitanguy em Fernando de Noronha tenham sido muito bem sucedidas e de grande valia em vários aspectos. Alcançamos um número significativo de consultas e cirurgias realizadas, proporcionamos agilidade na resolução de casos, efetuando o tratamento cirúrgico logo a seguir do diagnóstico, e promovemos a Cirurgia Plástica em um lugar carente desta especialidade.

Além do mais, este projeto representou uma economia evidente para o estado em termos de gastos com saúde pública e uma inestimável experiência de vida a todos aqueles que formaram estas equipes. A possibilidade de ajudar a população com as nossas habilidades e conhecimentos só nos orgulha e enaltece ainda mais a tradição de nossa escola.

\section{Correspondência para:}

Pablo Maricevich

Av. Ataulfo de Paiva, 27 - apto. 704 - Leblon - Rio de Janeiro, RJ, Brasil - CEP 22440-032

E-mail: jpmaricevich@hotmail.com 